\section{(6) OPEN ACCESS}

Department of Ophthalmology, Hôpital Lariboisière, Université Paris Diderot - Sorbonne Paris Cité, AP-HP, Paris, France

\section{Correspondence to \\ Professor Ramin Tadayoni, Service d'Ophtalmologie, Hôpital Lariboisière, 2, Rue Ambroise Paré, Paris 75010 France; \\ ramin.tadayoni@lrb.aphp.fr}

$\mathrm{RT}$ and IS contributed equally.

This paper was presented at the Association for Research in Vision and Ophthalmology Annual Meeting in May, 2011.

Accepted 23 September 2012 Published Online First 17 October 2012

\title{
Decreased retinal sensitivity after internal limiting membrane peeling for macular hole surgery
}

\author{
Ramin Tadayoni, Ivana Svorenova, Ali Erginay, Alain Gaudric, Pascale Massin
}

\begin{abstract}
Aims To compare the retinal sensitivity and frequency of microscotomas found by spectral domain optical coherence tomography (SD-OCT) combined with scanning laser ophthalmoscopy (SLO) microperimetry after idiopathic macular hole closure, in eyes that underwent internal limiting membrane (ILM) peeling and eyes that did not.
\end{abstract}

Methods This was a retrospective, non-randomised, comparative study. Combined SD-OCT and SLO microperimetry was performed in 16 consecutive eyes after closure of an idiopathic macular hole. A customised microperimetry pattern with 29 measurement points was used. The ILM was peeled in $8 / 16$ eyes. The main outcome measure was mean retinal sensitivity.

Results Mean retinal sensitivity (in $\mathrm{dB}$ ) was lower after peeling: $9.80 \pm 2.35 \mathrm{~dB}$ with peeling versus $13.19 \pm 2.92$ without $(p=0.0209)$. Postoperative microscotomas were significantly more frequent after ILM peeling: 11.3 \pm 6.6 points with retinal sensitivity below $10 \mathrm{~dB}$ in eyes that underwent peeling versus $2.9 \pm 4.6$ in those that did not $(p=0.0093)$.

Conclusions These results suggest that ILM peeling may reduce retinal sensitivity, and significantly increase the incidence of microscotomas. Until a prospective trial confirming or not these results, it seems justified to avoid peeling the ILM when its potential benefit seems minor or unproved, and when peeling is carried out, to limit the surface peeled to the bare minimum.

\section{INTRODUCTION}

Peeling of the internal limiting membrane (ILM) of the retina has proved beneficial for increasing the anatomic success rate of macular hole $(\mathrm{MH})$ surgery. ${ }^{1-3}$ This procedure has become popular, and many surgeons perform extensive ILM peeling in all cases of $\mathrm{MH}$ surgery and even for other unproved indications. However, as a few studies have also shown a high closure rate for small $\mathrm{MH}$ $(\leq 400 \mu \mathrm{m})$ without ILM peeling, probably due to other surgical refinements, ${ }^{4-7}$ there is still debate among vitreoretinal surgeons about whether and when to peel the ILM in MH cases. Whether peeling should be extended to the treatment of all $\mathrm{MH}$ and be widely used for unproved indications depends on its possible side effects, in particular its effects on visual function. Although the wish to achieve anatomic success is usually the main reason for using a surgical technique like ILM peeling, functional outcomes should also be considered in the risk-benefit analysis. ILM peeling has been shown to lead to small but noticeable anatomic changes in the peeled area of the retina, including causing the retina to have the appearance of a dissociated optic nerve fibre layer
(DONFL). ${ }^{8-9}$ However, whether peeling also induces functional deterioration of the retina remains controversial. $^{9-11}$

While visual acuity (VA) depends solely on the sensitivity of the centre of the fovea, microperimetry testing of the retina measures retinal sensitivity at specific points over a larger surface and reveals relative or absolute microscotomas. Reduced retinal sensitivity and the presence of paracentral microscotomas may cause visual discomfort despite good $\mathrm{VA}$, as reported by some patients after $\mathrm{MH}$ surgery. The purpose of this study was therefore to investigate, in eyes that had undergone ILM peeling and eyes that had not, the retinal sensitivity and frequency of these microscotomas after $\mathrm{MH}$ closure, using a new type of scanning laser ophthalmoscopy (SLO) microperimetry combined with spectral domain optical coherence tomography (SD-OCT).

\section{MATERIALS AND METHODS \\ Patients}

In a retrospective, non-randomised, comparative study, 16 eyes of 16 consecutive patients who had experienced idiopathic $\mathrm{MH}$ closure were evaluated in our department. Patients were informed about the use of their data for this retrospective study. Eight eyes underwent ILM peeling, and eight did not (the latter included one eye with a $\mathrm{MH}$ which closed spontaneously). The characteristics of the two groups are shown in table 1. None of the eyes had any other retinal disorders or had undergone previous retinal surgery. Table 2 shows the characteristics of their $\mathrm{MH}$.

\section{Surgical technique}

The surgical technique used was a standard 3-port pars plana vitrectomy. If the posterior hyaloid was still attached to the optic disc, its detachment was induced. When present, the epiretinal membrane around the hole was removed. The ILM was visualised in five eyes with Brilliant Blue G (Geuder, Heidelberg, Germany), in two eyes with $0.5 \%$ trypan blue (MembraneBlue, DORC International, Zuidland, The Netherlands) and in one eye with triamcinolone acetonide (Kenacort Retard, BristolMyers Squibb, Princeton, New Jersey, USA). The ILM was peeled off eight eyes with forceps in an area of $\geq 1$ disc diameter around the $\mathrm{MH}$. Fluid-air exchange and intraocular gas tamponade with C2F6 were performed.

\section{Data}

At1-12 months after $\mathrm{MH}$ closure, patients underwent clinical examination, colour fundus photography, retinal $3 \mathrm{D}$ optical coherence tomography (OCT) (confirming the closure of the $\mathrm{MH}$ ) and 
Table 1 Characteristics of patients with and without ILM peeling

\begin{tabular}{lllc}
\hline & ILM peeling $(\mathbf{n}=8)$ & No peeling $(\mathbf{n}=8)$ & $\mathbf{p}$ Value \\
\hline Age (years) & $64.6 \pm 5.6$ & $71.1 \pm 5.5$ & 0.0352 \\
Gender (male/female) & $3 / 5$ & $4 / 4$ & $>0.999$ \\
Preoperative BCVA (log MAR) & $0.97 \pm 0.56$ & $0.54 \pm 0.20$ & 0.0582 \\
Postoperative BCVA (log MAR) & $0.57 \pm 0.28$ & $0.26 \pm 0.16$ & 0.0139 \\
Lens status (phakic/OL) & $4 / 4$ & $2 / 6$ & 0.3147 \\
\hline
\end{tabular}

Results are means \pm SD.

BCVA, best corrected visual acuity; ILM, internal limiting membrane; IOL, intraocular lens; log MAR, logarithm of the minimum angle of resolution.

combined spectral OCT/SLO topography and microperimetry (OPKO/OTI, Miami, Florida, USA). The latter device allows the testing of retinal sensitivity and observation of the fundus by SLO and SD-OCT. An automatic fundus eye tracking system enabled accurate projection of the stimulus, always onto the same point on the retina, according to retinal vessel alignment. In brief, the system acquires images at 4-8 frames per second. A landmark area, automatically proposed or selected by the operator, is tracked. Before each stimulus is shown, the pattern is moved, to reflect the current position of the eye, and the appropriate location on the SLO is stimulated. Compared to previous devices, this SLO-microperimetry imaging allows the accurate assessment of retinal sensitivity at specific points on the retina even if the patient's fixation is relatively poor.

For 14 of the 16 patients, a customised pattern was used (figure 1) in $9^{\circ}$ of the visual field, with 29 spots (21 central and 8 mid ring). This pattern, with its dense location of 29 spots, gives a suitable evaluation of macular sensitivity and enables the detection even of small visual field defects in the macular area. We used a Goldman size II stimulus with a duration of $200 \mathrm{~ms}$ and an interval between stimuli of $2000 \mathrm{~ms}$. For the remaining two patients, the Polar 3 pattern with 28 spots (4 central, 12 mid ring and 12 outer ring) was used. The size and duration of the stimuli and the interval between them were similar to those of the customised pattern. Note that the $\mathrm{MH}$ sequelae might potentially have affected the single central point measurement when other measurements are supposed to be in normal retina areas.

On the basis of the microperimetry findings, we evaluated mean retinal sensitivity (primary outcome), the presence of

Table 2 Characteristics of macular holes in eyes with and without ILM peeling

\begin{tabular}{llll}
\hline & ILM peeling $(\mathbf{n}=8)$ & No peeling $(\mathbf{n = 8})$ & p Value \\
\hline $\begin{array}{l}\text { Preoperative size of the } \mathrm{MH}(\mu \mathrm{m}) \\
\text { MH diameter } \leq 400 \mu \mathrm{m}(\mathrm{n})\end{array}$ & $492 \pm 195$ & $270 \pm 120$ & 0.0669 \\
MH diameter $>400 \mu \mathrm{m}(\mathrm{n})$ & 5 & 6 & \\
VM stage of the $\mathrm{MH}^{*}$ & & 2 & 0.2620 \\
Stage 1B & - & 1 & \\
Stage 2 & - & 1 & \\
Stage 3 & 6 & 3 & \\
Stage 4 & 2 & 1 &
\end{tabular}

${ }^{*}$ Optical coherence tomography staging: stage $1 \mathrm{~B}$, impending macular hole, which opens the outer retinal layer; stage 2, full thickness idiopathic macular hole with vitreous attached to the edge of the hole through the pseudo operculum; stage 3 , vitreous detached from the macula but attached to the optic disc; stage 4, complete posterior vitreous detachment. ${ }^{16}$

ILM, internal limiting membrane; MH, macular hole; VM, vitreomacular. absolute or relative microscotomas with a retinal sensitivity of less than $10 \mathrm{~dB}$, and the average lowest $\mathrm{dB}$, in eyes that underwent ILM peeling and those that did not.

\section{Statistical analysis}

Results are expressed as means \pm SD. The Mann-Whitney nonparametric test was used to compare the statistical distribution of the parameters measured. Fisher's exact test was used for categorical variables.

\section{RESULTS}

The average retinal sensitivity (in $\mathrm{dB}$ ) of the macular area was significantly lower after peeling: $9.80 \pm 2.35 \mathrm{~dB}$ in peeled eyes versus $13.19 \pm 2.92 \mathrm{~dB}$ in unpeeled eyes $(p=0.0209)$. The least sensitive point in the pattern, reflecting the depth of the retinal sensitivity decrease, had an average value of $1.38 \pm 3.16 \mathrm{~dB}$ in the group with ILM peeling, versus $7.25 \pm 4.83 \mathrm{~dB}$ in the control group with no peeling $(p=0.0243)$.

A central absolute microscotoma $(0 \mathrm{~dB})$ was found in two patients in the group with no peeling and in one patient with peeling. On OCT, all the central absolute microscotomas were associated with disruption of the inner/outer photoreceptor segment junction in the foveal centre, as a consequence of the $\mathrm{MH}$.

Paracentral absolute microscotomas (outside the area affected by the initial disease, that is, the $\mathrm{MH}$ ) were only found in eyes whose ILM was peeled off during surgery ( $5 / 8$ eyes vs $0 / 8$ in the control group, $p=0.0256$ ): in four of these five eyes, we found one paracentral absolute microscotoma, and in one eye, four such microscotomas. Postoperative relative microscotomas were also significantly more frequent after ILM peeling: $11.3 \pm 6.6$ points with retinal sensitivity below $10 \mathrm{~dB}$ in eyes that underwent peeling versus $2.9 \pm 4.6$ in those that did not $(p=0.0093$, table 3$)$.

\section{DISCUSSION}

As far as we know, this is the first study to quantify retinal sensitivity after ILM peeling using the accurate technique of OCT/SLO eye tracking microperimetry. A new pattern was used to ensure adequate density of the measurement points in the macular area. As MH only affects the centre of the macula, the measurement points, except for the central point, were located in the formerly normal area of the retina. Therefore, comparison of the data for eyes whose $\mathrm{MH}$ was closed and which underwent ILM peeling to the data for eyes without peeling gives a strong indication of the effect of peeling on the normal retina. We found that mean retinal sensitivity was significantly lower (in $\mathrm{dB}$ ) in eyes that underwent peeling than in those that did not. Postoperative microscotomas were also significantly more frequent in eyes that had undergone peeling.

Although, as stated in the Introduction section, a desire to increase the anatomic success rate has been the main motive for including ILM peeling in the treatment of several diseases, including $\mathrm{MH}$, functional outcomes such as postoperative scotomas and reduced retinal sensitivities should also be considered in the risk-benefit analysis. Even if there are no intraoperative complications, ILM peeling can cause anatomic changes in the retina, such as a DONFL appearance of the fundus, which has frequently been reported. ${ }^{8-11}$ The DONFL appearance consists of numerous arcuate striae slightly darker than the surrounding retina. On OCT it is visible in the form of defects in the optic nerve fibre layer. ${ }^{12}$ Whether or not the presence of a DONFL adversely affects retinal function is still controversial. In a study using the conventional Humphrey perimetry 10-12 

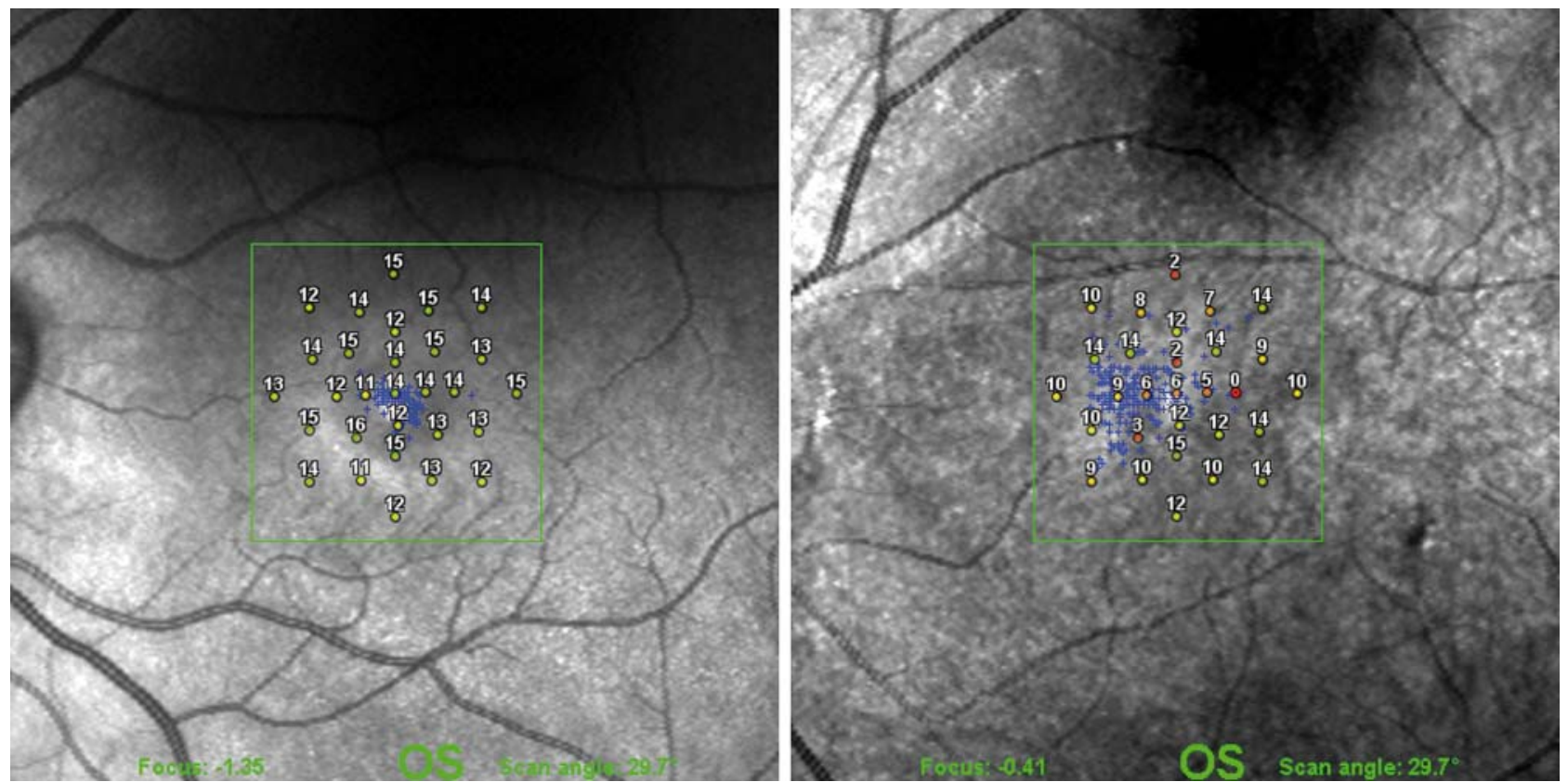

Figure 1 Scanning laser ophthalmoscope microperimetry image with superimposed location of the patient's fixation (blue crosses) after idiopathic macular hole surgery. (Left) At 1 month after surgery without internal limiting membrane (ILM) peeling: normal retinal sensitivity and no deep microscotomas in the central $9^{\circ}$ of the visual field in this eye. (Right) At 2 months after surgery with ILM peeling: decreased mean retinal sensitivity, deep absolute microscotoma and relative microscotomas in the central $9^{\circ}$ of the retina of this eye. This figure is only reproduced in colour in the online version.

program, no scotomas were detected in any of 20 eyes which had undergone idiopathic $\mathrm{MH}$ surgery with ILM peeling. ${ }^{9}$ The results of microperimetry evaluations are conflicting: a few authors found no changes after peeling, ${ }^{13-14}$ while a few others showed the presence of microscotomas. ${ }^{10-11}$ This discrepancy is partly due to the different technologies used. VA, standard Humphrey visual field testing and even some microperimetry techniques might miss some of the subtle defects that the present SD-OCT/SLO approach detected. As stated in the Methods section, the new OCT/SLO microperimetry device used in our study to test retinal sensitivity is, with its OCT and eye tracking system, one of the most precise microperimetry methods available. Moreover, we used a customised pattern with a high density of central points, to obtain dense coverage of the central area of the macular region. With this new method, mean macular sensitivity was found to be lower by about $3.4 \mathrm{~dB}$ in eyes with ILM peeling than in those without. It should be remembered that the decibel $(\mathrm{dB})$ is a logarithmic

Table 3 Microperimetry measurements after macular hole closure of eyes with and without ILM peeling

\begin{tabular}{|c|c|c|c|}
\hline & $\begin{array}{l}\text { ILM peeling } \\
(\mathrm{n}=8)\end{array}$ & $\begin{array}{l}\text { No peeling } \\
(\mathrm{n}=8)\end{array}$ & p Value \\
\hline Retinal sensitivity (dB) & $9.80 \pm 2.35$ & $13.19 \pm 2.92$ & 0.0209 \\
\hline Sensitivity of the lowest point $(\mathrm{dB})$ & $1.38 \pm 3.16$ & $7.25 \pm 4.83$ & 0.0243 \\
\hline Microscotomas* (n/eye) & $11.3 \pm 6.6$ & $2.9 \pm 4.6$ & 0.0093 \\
\hline $\begin{array}{l}\text { Presence of absolute paracentral } \\
\text { microscotomas (eyes) }\end{array}$ & 5 & 0 & 0.0256 \\
\hline $\begin{array}{l}\text { Presence of absolute central } \\
\text { microscotomas (eyes) }\end{array}$ & 1 & 2 & $>0.999$ \\
\hline
\end{tabular}

Results are means \pm SD.

${ }^{*}$ Points with retinal sensitivity $<10 \mathrm{~dB}$.

ILM, internal limiting membrane. unit that indicates the ratio of a physical quantity, and that a $3 \mathrm{~dB}$ change is a change in power ratio by approximately a factor of two. In other words, after ILM peeling, the retina needed more than twice as much light to see the spot as before peeling.

Five of the eight eyes that underwent peeling exhibited at least one paracentral absolute microscotoma (one eye exhibited four). Note that the presence of these microscotomas was revealed by 29 pinpoint measurements. Consequently, these eyes may have had many more paracentral microscotomas than were revealed with the present number of measurement points. These absolute microscotomas seem strongly correlated to ILM peeling, as they were not found in any of the eyes that did not undergo peeling.

Moreover, in eyes that underwent peeling, more than a third of the points measured had a sensitivity of $<10 \mathrm{~dB}$, compared to about one in 10 of the points for unpeeled eyes. All these abnormalities may reduce the quality of vision, even if they do not actually reduce VA. These results for microscotomas are in line with those reported in two studies using the Rodenstock SLO-105 device for microperimetry and covering the central $8-10^{\circ}$ of the visual field with a Goldmann II size stimulus. ${ }^{10-11}$ In one of these studies, ${ }^{10}$ paracentral scotomas were found in $56 \%$ of the eyes, which had undergone vitrectomy with ILM removal for idiopathic $\mathrm{MH}$ without the use of any dye. In an earlier study of eyes that underwent surgery for idiopathic $\mathrm{MH}$, also with ILM removal, ${ }^{11}$ the same authors had shown the occurrence of paracentral scotomas in areas which had been tested and found to be normal before surgery, but which thereafter exhibited a DONFL appearance. ${ }^{11}$

The cause of the development of microscotomas after peeling has still not been established. The direct trauma caused by the forceps when gripping the ILM may not be the main cause of all these microscotomas, because first, operations were performed by experienced surgeons (RT, AG, PM) accustomed 
to exercise caution when peeling off the ILM, and second, because the deterioration in retinal sensitivity was too diffuse to be explained by a direct retinal trauma. An effect of dyes, which were only used for peeled eyes, cannot be completely excluded, but as the three different dyes used did not include indocyanine green (ICG), the only dye that has been demonstrated to have a toxic effect on ganglion cells, this hypothesis seems unlikely. Retinal sensitivity deterioration and microscotomas might, like the development of a DONFL appearance, be due to deterioration of the retina, especially of the Müller cells, whose endfeet are closely connected to the ILM and may be affected by ILM peeling. ${ }^{15}$ Deterioration of other cells is also possible, either directly, due to the stretching caused by the peeling, or indirectly, due to Müller cell deterioration.

As the present work is a retrospective study, it has some limitations, including the different periods that elapsed between surgery and the postoperative examination, and the different sizes of the $\mathrm{MH}$. However, the preoperative characteristics of the two groups of patients did not exhibit any differences that might have caused postoperative differences in retinal sensitivity (tables 1 and 2). In this study, there was also a difference between the maximal best corrected visual acuity (BCVA) reached in the eyes that underwent ILM peeling and those that did not. BCVA was significantly better (lower logarithm of the minimum angle of resolution (log MAR)) in the unpeeled than the peeled group, probably because, in our department, we usually peel off the ILM for $\mathrm{MH} \geq 400 \mu \mathrm{m}$, which have a lower preoperative $\mathrm{VA}$, and these larger $\mathrm{MH}$ may therefore have a lower maximal postoperative VA than eyes with a smaller $\mathrm{MH}$, whose ILM is not usually peeled off. However, the size of the $\mathrm{MH}$ did not affect retinal sensitivity outside the hole, or the presence of paracentral microscotomas after ILM peeling, because the area in which paracentral microscotomas were present was much larger than that of the $\mathrm{MH}$. As in this series we did not have eyes with ILM peeled without staining or operated on by less experience surgeons, the effects of these factors could not be studied. Indeed, less experience and lack of staining may cause more scotomas due to greater difficulty grasping the ILM and direct trauma to the retina.

In the absence of a prospective trial confirming the present results, the findings of this study should be evaluated and compared with the benefit of ILM peeling. From this point of view, the demonstrated benefit of peeling in increasing the postoperative closure rate of large surgically treated $\mathrm{MH}$ surpasses the risks. However, in many unproved indications for ILM peeling, or even in small $\mathrm{MH}$ for which it seems at least possible to achieve a high closure rate with procedures other than ILM peeling, ${ }^{4-7}$ the probable reduction in retinal sensitivity after peeling should be taken into account. In these cases, ILM peeling should, in our opinion, be reserved for rare cases of first surgery failure or of recurrence, as this strategy would ensure the best quality of vision for the remaining large majority of these patients.

In summary, ILM peeling may reduce retinal sensitivity and cause postoperative microscotomas. Our retrospective study justifies a larger randomised study to confirm the present results. Such confirmation should lead to more rigorous selection of eyes to undergo ILM peeling. In the meantime, the present results justify the avoidance of such peeling for unproved indications, if its potential benefit seems small. However, if peeling is decided upon, the surface peeled should be limited to the bare minimum.

Contributors RT: conception and design, analysis and interpretation of data, revision of the manuscript and final approval; IS: data acquisition, interpretation of data, drafting the manuscript; AE: acquisition and interpretation of data; AG: design, interpretation of data, revision of the manuscript; PM: interpretation of data, revision of the manuscript.

\section{Competing interests None.}

Provenance and peer review Not commissioned; externally peer reviewed.

\section{REFERENCES}

1. Lois $\mathbf{N}$, Burr JM, Norrie $\mathrm{J}$, et al. Internal limiting membrane peeling versus no peeling for idiopathic full thickness macular hole: A pragmatic randomised controlled trial. Invest Ophthalmol Vis Sci 2011;52:1586-92.

2. Christensen UC. Value of internal limiting membrane peeling in surgery for idiopathic macular hole and the correlation between function and retinal morphology. Acta Ophthalmol 2009;87:Thesis 2:1-23.

3. Kwok AK, Lai TY, Wong VW. Idiopathic macular hole surgery in Chinese patients: a randomised study to compare indocyanine green-assisted internal limiting membrane peeling with no internal limiting membrane peeling. Hong Kong Med $J$ 2005;11:259-66

4. Tadayoni R, Gaudric A, Haouchine B, et al. Relationship between macular hole size and the potential benefit of internal limiting membrane peeling. $\mathrm{Br} \mathrm{J}$ Ophthalmol 2006;90:1239-41.

5. Tadayoni R, Vicaut E, Devin F, et al. A randomized controlled trial of alleviated positioning after small macular hole surgery. Ophthalmology 2011;118:150-5.

6. Ullrich S, Haritoglou C, Gass C, et al. Macular hole size as a prognostic factor in macular hole surgery. Br J Ophthalmol 2002;86:390-3.

7. Ip MS, Baker BJ, Duker JS, et al. Anatomical outcomes of surgery for idiopathic macular hole as determined by optical coherence tomography. Arch Ophthalmol 2002;120:29-35.

8. Tadayoni R, Paques M, Massin P, et al. Dissociated optic nerve fiber layer appearance of the fundus after idiopathic epiretinal membrane removal. Ophthalmology 2001;108:2279-83.

9. Ito $\mathbf{Y}$, Terasaki $H$, Takahashi $A$, et al. Dissociated optic nerve fiber layer appearance after internal limiting membrane peeling for idiopathic macular holes. Ophthalmology 2005;112:1415-20.

10. Haritoglou C, Gass CA, Schaumberger M, et al. Long-term follow-up after macular hole surgery with internal limiting membrane peeling. Am J Ophthalmol 2002:134:661-6.

11. Haritoglou CH, Ehrt 0, Gass CA. Paracentral scotomata: a new finding after vitrectomy for idiopathic macular hole. Br J Ophthalmol 2001;85:231-3.

12. Kishimoto H, Kusuhara $S$, Matsumiya W, et al. Retinal surface imaging provided by Cirrus high-definition optical coherence tomography prominently visualizes a dissociated optic nerve fiber layer appearance after macular hole surgery. Int Ophthalmol 2011;31:385-92.

13. Mitamura Y, Ohtsuka K. Relationship of dissociated optic nerve fiber layer appearance to internal limiting membrane peeling. Ophthalmology 2005:112:1766-70.

14. Imai H, Ohta K. Microperimetric determination of retinal sensitivity in areas of dissociated optic nerve fiber layer following internal limiting membrane peeling. Jpn J Ophthalmol 2010;54:435-40.

15. Haritoglou C, Schumann R, Reiniger I, et al. Evaluation of the internal limiting membrane after conventional peeling during macular hole surgery. Retina 2006;26:21-4.

16. Haouchine B, Massin P, Gaudric A. Foveal pseudocyst as the first step in macular hole formation: a prospective study by optical coherence tomography. Ophthalmology 2001;108:15-22. 